\title{
Lactate Clearance at 6 Hours of Admission as a Predictor of Mortality in Critically Ill Children
}

\section{Azza A. Moustafa ${ }^{1}$, Hadeer M. Hassouna ${ }^{1}$, Abeer El-Hadidi ${ }^{2}$, Mona El-Najar ${ }^{1}$ ${ }^{1}$ Pediatrics Department, Faculty of Medicine, Alexandria University, ${ }^{2}$ Clinical Pathology Department, Faculty of Medicine, Alexandria University, Egypt}

\section{INIRODUCIION}

- The concentrations of arterial lactate represent the balance between lactate production and clearance. Hyperlactatemia occurs when production exceeds clearance, which has been confirmed to be associated with worse clinical outcomes in critically ill patients. ${ }^{(1)}$ In critically ill patients, failure of oxygen delivery to meet oxygen demand results in tissue hypoxia and increases anaerobic metabolism. Elevated serum lactate levels reflect the anaerobic metabolism related to cellular hypoxia and are thought to be an important marker of impaired tissue perfusion. (2) Unresolved global tissue hypoxia, as indicated by inadequate lactate clearance, is associated with multi-organ dysfunction and increased mortality during the early phase of resuscitation. $(2,3)$$$
\text { AIM OF THE WORK }
$$

- To evaluate the use of lactate clearance at 6 hours of admission to Pediatric Intensive Care Unit as a predictor of mortality in critically ill children.$$
\text { METHODS }
$$

- A prospective observational study conducted in PICU of a tertiary care teaching hospital over 6 months. Lactate levels were measured in arterial blood samples of 76 patients at the time of admission and six hours later. According to lactate clearance after 6 hours, patients were divided into group A (lactate clearance $>0 \%$ ) and group B (lactate clearance $\leq 0 \%$ ). Routine care and investigations were performed and Pediatric Index of Mortality 2 (PIM 2) score was calculated.

\section{RESULTS}

- There were 54 patients in group $A(71 \%)$ and 22 patients in group B (29\%). Using Kaplan-Meier survival curve group A had significant higher overall survival than group B $(p<0.001)$. Mean PIM2 score and risk of mortality was significantly higher in group $B(p=0.001)$, while there was no significant difference in the duration of PICU admission between the two groups ( $p=$ 0.657). Using the ROC curves, the area under the ROC curves (AUC) indicated a strong significant predictive power for the PIM2 score, which represents the largest AUC $(0.935, p<0.001)$ followed by that of lactate clearance after 6 hours of admission $(0.766, p<0.001)$. Lactate clearance after 6 hours of admission predicted mortality with sensitivity of $70.8 \%$, specificity of $90.4 \%$, Positive predicting value of $77.3 \%$, and negative predicting value of $87 \%$. Using logistic regression model, both lactate clearance after 6 hours and PIM2 score had independent prognostic significance as regard fate. $(p=0.030,0.001$ respectively).

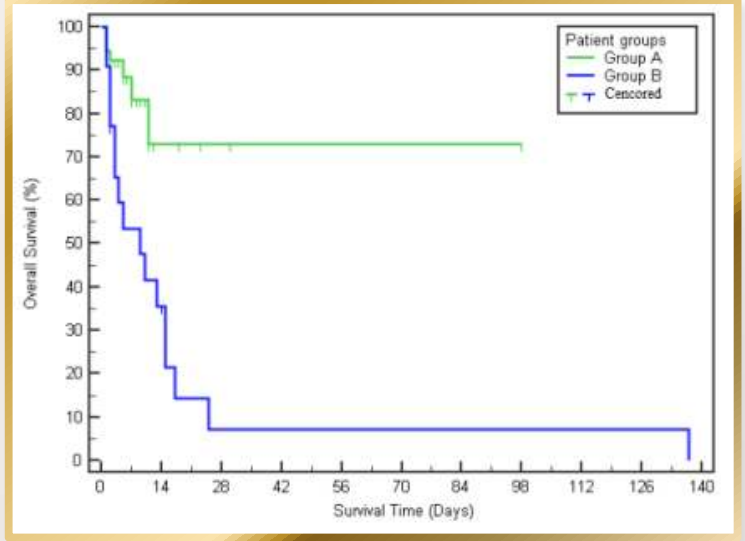

Kaplan Meier survival curve of the studied groups

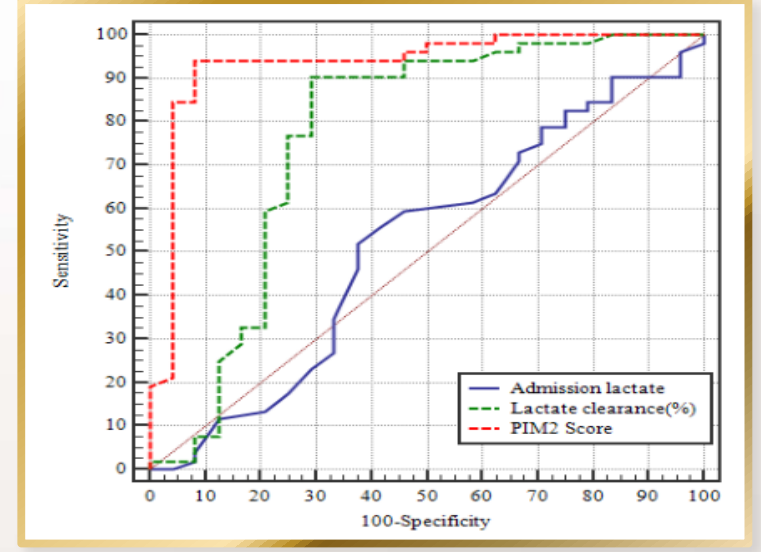

ROC curve for lactate on admission, lactate clearance and PIM2 score

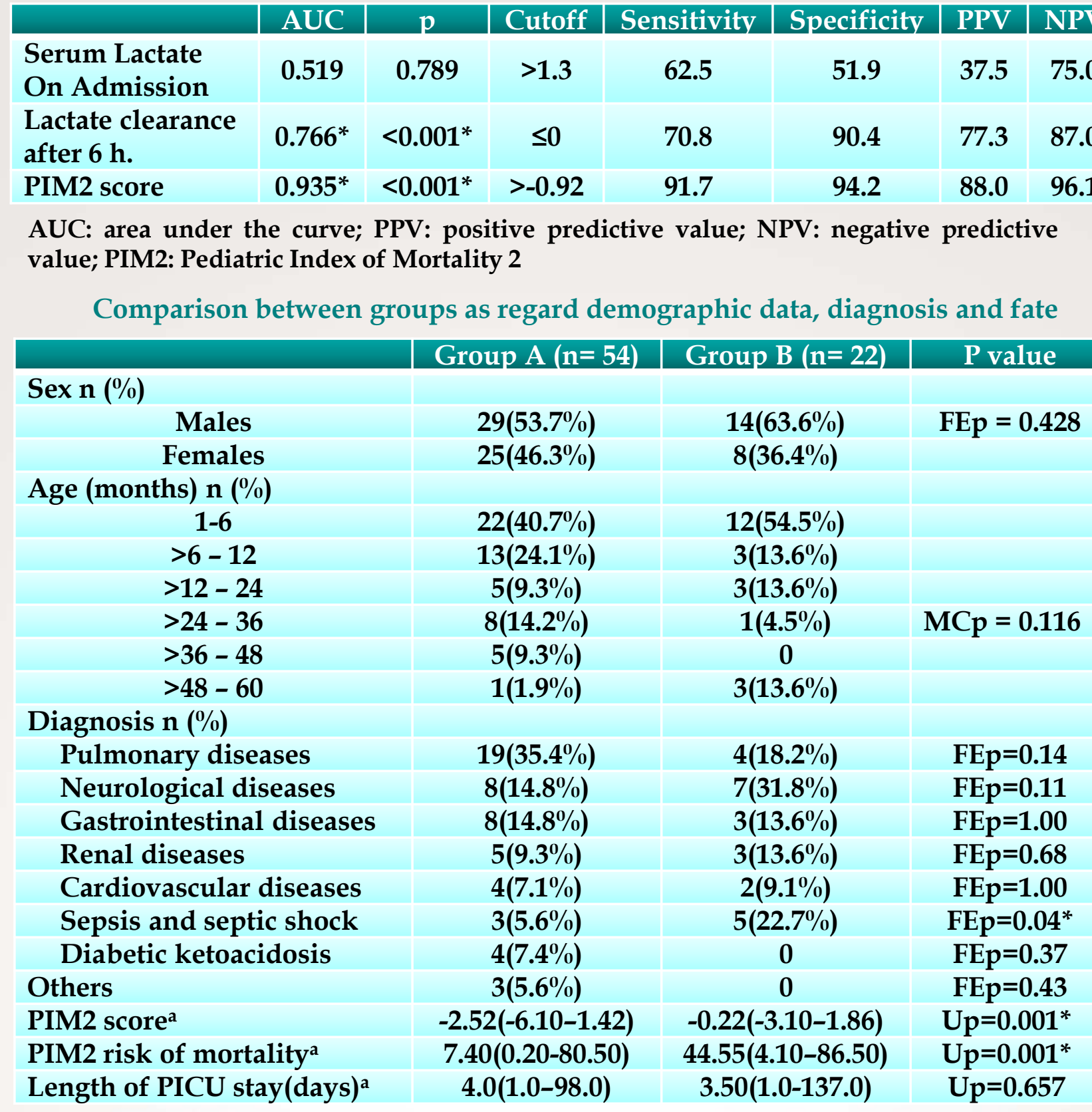

Comparison between groups as regard laboratory findings
White Blood Cells $\left(\times 10^{3} / \mu \mathrm{L}\right)^{\mathrm{a}}$

Hemoglobin (g/dl)

Platelets $\left(\times 10^{3} / \mu \mathrm{L}\right)^{\mathrm{a}}$

$\operatorname{ALT}(\mathrm{U} / \mathrm{L})^{\mathrm{a}}$

AST (U/L)

Serum Albumin $(\mathrm{g} / \mathrm{dl})^{\mathrm{b}}$

Total Bilirubin (mo/dL)

Direct Bilirubin $(\mathrm{mg} / \mathrm{dL})^{\mathrm{b}}$

Prothrombin time (seconds)

Partial thromboplastin time (seconds)

International normalized ratio (INR)

Blood Urea Nitrogen $(\mathrm{mg} / \mathrm{dL})^{\mathrm{a}}$

Serum Creatinine $(\mathrm{mg} / \mathrm{dL})^{\mathrm{a}}$

C-Reactive protein $(\mathrm{mg} / \mathrm{mL})^{\mathrm{a}}$

Serum Sodium $(\mathrm{mmol} / \mathrm{L})^{\mathrm{b}}$

Serum Potassium $(\mathrm{mmol} / \mathrm{L})^{\mathrm{b}}$

Serum Calcium(mg/dL)

Serum Phosphorus $(\mathrm{mg} / \mathrm{dL})^{\mathrm{a}}$

Alkaline phosphatase $(\mathrm{U} / \mathrm{L})^{\mathrm{a}}$

Admission Lactate $(\mathrm{mmol} / \mathrm{L})^{\mathrm{a}}$

Lactate after $6 \mathrm{hrs}(\mathrm{mmol} / \mathrm{L})^{\mathrm{a}}$

Admission Lactate/pyruvate ratio ${ }^{\mathrm{a}}$

Lactate/pyruvate ratio after 6 hrs

$t p=p$ value for Student $t$-test $*$ : Statistically significant at $p \leq 0.05$

\begin{tabular}{|c|c|c|}
\hline & & \\
\hline 3.15 & $15.49(3.40-99.10)$ & ${ }^{\mathrm{u}} \mathrm{p}=0.4$ \\
\hline 6.50 & $2.80 \pm 12.7$ & ${ }^{t} p=0.30$ \\
\hline $6.50(0.4$ & $365(12-692)$ & ${ }^{u} p=0.29$ \\
\hline $0.31(9-6651)$ & $28(13-6$ & ${ }^{\mathrm{u}} \mathrm{p}=0.61$ \\
\hline $8.50(10$ & $45(18-$ & \\
\hline $3.06=$ & $2.59=$ & ${ }^{t} p=0.026^{*}$ \\
\hline $0.34(0.10$ & $0.32(0.1$ & ${ }^{\mathrm{u}} \mathrm{p}=0.83$ \\
\hline $0.10(($ & & ${ }^{\mathrm{u}} \mathrm{p}=0.67$ \\
\hline 12.8(9. & 1 & ${ }^{\mathrm{u}} \mathrm{p}=0.12$ \\
\hline $33.2(20-1)$ & .450 & ${ }^{\mathrm{u}} \mathrm{p}=0.48$ \\
\hline 1.18(1. & 04 & ${ }^{\mathrm{u}} \mathrm{p}=\mathbf{0 . 1 8}$ \\
\hline 120 & & ${ }^{\mathrm{u}} \mathrm{p}=$ \\
\hline $0.47(0$ & 0.44 & ${ }^{\mathrm{u}} \mathrm{p}=0.709$ \\
\hline $5(0.0-26$ & 12.4( & ${ }^{u} p=0.146$ \\
\hline 140.4 & 144. & ${ }^{t} p=0.286$ \\
\hline 4.06 & 3.43 & ${ }^{\mathrm{t}} \mathrm{P}=0.007^{*}$ \\
\hline & & ${ }^{t} \mathrm{p}=0$ \\
\hline $4.60(2$. & $.5(1$ & ${ }^{u} p=0.136$ \\
\hline 169.5( & 156.5 & ${ }^{\mathrm{u}} \mathrm{p}=0.689$ \\
\hline $1.60(0.50-19.0)$ & $1.20(0$ & ${ }^{u} p=0.010^{*}$ \\
\hline $0.90(0$ & 1.95 & ${ }^{u} p=0.001^{*}$ \\
\hline & & $p=0.025^{*}$ \\
\hline $15.0(4.0-230.0)$ & $3.50(9.0-229.0)$ & ${ }^{u} p=0.00$ \\
\hline
\end{tabular}

Mann Whitney test,

\section{LONCLUSION}

- Lactate clearance is fundamental in prediction of mortality; as early and effective lactate clearance is associated with decreased mortality. Serial evaluations of serum lactate may have greater value over measuring serum lactate only at the time of presentation. It was found that PIM2 score still a better predictor of mortality.

\section{REFERENCES}

1. Marik P, Bellomo R. Lactate clearance as a target of therapy in sepsis: a flawed paradigm. OA Crit Care. 2013; 1(3).

2. Nguyen HB, Rivers EP, Knoblich BP, Jacobsen G, Muzzin A, Ressler JA, et al. Early lactate clearance is associated with improved outcome in severe sepsis and septic shock. Critical Care Medicine. 2004;32 (8):1637-42.

3. Kim YA, Ha E-J, Jhang WK, Park SJ. Early blood lactate area as a prognostic marker in pediatric septic shock. Intensive Care Medicine. 2013; 39 (10):1818-23. 